\title{
Publication and Interpretation of Clinical Trial Results: The Need for Caution
}

\author{
J. F. Thompson, MD ${ }^{1,2}$, A. Hong, MBBS, PhD ${ }^{1,2}$, and G. Fogarty, MBBS ${ }^{1,2}$ \\ ${ }^{1}$ Sydney Medical School, The University of Sydney, Sydney, NSW, Australia; ${ }^{2}$ Melanoma Institute Australia, \\ North Sydney, NSW, Australia
}

When the results of a clinical trial are published, modern information technology ensures that news of the trial outcome is disseminated rapidly to the medical community worldwide, and often to the general public as well, through the media. However, there is always a danger that physicians, patients, and the general public may be inappropriately influenced by the "spin" that is put on what they read or hear, without subjecting the trial report to critical evaluation. The adverse and inappropriate effects that the publication of clinical trial results can have on medical practice were discussed in an editorial by Kaplan, who pointed out the heavy responsibilities that fall on the shoulders of reviewers and journal editors to ensure that trials do not have serious design flaws and that their results are clearly and accurately reported. ${ }^{1}$

The publication in February 2011 of the results of a large, randomized study assessing the value of completion lymph node dissection (CLND) in sentinel node (SN)positive breast cancer patients illustrates how problems of misinterpretation can arise. The trial report was published in the Journal of the American Medical Association (JAMA), a leading international medical journal, and it was featured on the front page of the New York Times shortly afterwards. ${ }^{2,3}$ The title of the report in JAMA was "Axillary dissection versus no axillary dissection in women with invasive breast cancer and sentinel node metastasis: a randomized clinical trial." However, the dramatic headline in the New York Times read, "Lymph node study shakes pillar of breast cancer care," and there were similar

(C) Society of Surgical Oncology 2012

First Received: 14 February 2012;

Published Online: 5 April 2012

J. F. Thompson, MD

e-mail: john.thompson@melanoma.org.au alarmist headlines in other major newspapers around the world. As a result of this widespread press publicity, we have encountered patients with melanoma who are not willing to consider entry into another large clinical trial, this one assessing the value of CLND when metastatic melanoma has been found in a SN (MSLT-II-Clinicaltrials.gov identifier NCT00297895). These patients have become aware that in the trial report in JAMA, Giuliano and colleagues concluded that CLND is not necessary for breast cancer patients found to be SN-positive. The patients assume that the situation for SN-positive melanoma patients will be the same, not understanding that the two tumor types behave in quite different ways, and that in any case all the SN-positive patients with breast cancer enrolled in the trial received radiation therapy to the axilla, and most (96.5\%) also received systemic chemotherapy (known to be effective for breast cancer, but not for melanoma).

Another trial, published in a major medical journal in November 2009, also is having a major impact on clinical trial accrual in patients with metastatic melanoma, in this case those with cerebral metastases. ${ }^{4}$ The trial was reported by Chang and colleagues in Lancet Oncology and will be referred to hereafter as the Chang trial. ${ }^{4}$ It studied patients with cerebral metastases from cancers of various types who were treated with stereotactic radiosurgery (SRS) with or without whole brain radiotherapy (WBRT). The Chang trial was stopped early because one of six neurocognitive function assessment tools showed a detrimental result at 4 months. It is unknown whether this was a temporary or permanent effect of WBRT, because the trial was terminated before sufficient follow-up times had been achieved to assess long-term results.

There are many potential pitfalls in the design, conduct, and reporting of clinical trials, and the Chang trial appears to have had several flaws. ${ }^{5}$ Some of these have been detailed in published correspondence, but there are a 
number of additional, as yet unreported, criticisms. ${ }^{6-8}$ These include a discrepancy between the trial endpoints that were originally listed and those in the published report. ${ }^{9}$ Survival, local recurrence, and distant recurrence were designated as the primary endpoints to be investigated, but the final report looked primarily at toxicity, a textbook case of outcomes reporting bias. ${ }^{10}$ Another concern about the Chang trial is that the statistical basis for stopping the study reported in the article was the application of a Bayesian statistics approach, which indicated a $96 \%$ confidence that total recall at 4 months (as measured by the Revised Hopkins Verbal Learning Test) for the WBRT + SRS group was poorer than that of the SRSalone patients. However, the stated stopping rule in the protocol was that if the probability of (major) neurocognitive decline was at least $97.5 \%$ it would be necessary to stop the trial. Hence, the stopping boundary had not been met. Additionally, the amount of missing data was substantial (at 4 months only 11 of 28 and 20 of 30 subjects in the two arms of the trial were available for evaluation), casting serious doubt on the reliability of the estimates used to stop the study. It is unfortunate and disappointing that the Chang trial appears to have been stopped too early. It is possible that there may have been other factors that contributed to the stopping that were not reported in the publication. Even so, the Chang study in no way provides a definitive answer to the question of whether adjuvant WBRT after SRS is of value in the treatment of patients with brain metastases, especially those with melanoma.

Despite its serious shortcomings, the Chang trial is having a significant impact on clinical management and is already being quoted in the literature as showing that WBRT is detrimental. ${ }^{11}$ Sensational headlines in the medical press are likely to have contributed to the problem. One widely read medical newsletter, for example, ran an article entitled "Whole-brain radiation increases risk for cognitive decline in patients with brain metastases." 12 The article did not emphasize the important finding that after 1 year the patients who had received adjuvant WBRT after SRS had much higher local tumor control (100 vs. $67 \%$, $p=0.012$ ), much better distant brain tumor control (73 vs. $45 \%, p=0.02$ ), and much greater freedom from central nervous system recurrence ( 73 vs. $27 \%, p=0.0003$ ). An editorial published in the same issue of Lancet Oncology as the Chang trial was more objective and even-handed, and had a non-emotive title. It pointed out the results of a recent Japanese study involving patients with one to four brain metastases in whom those who had WBRT after SRS had greatly reduced CNS failures compared with SRS alone but, importantly for the present discussion, had neurocognitive outcomes that were equivalent at 1 year. ${ }^{13}$

It is clearly important to evaluate the role of adjuvant WBRT in patients with brain metastases as objectively and reliably as possible, and it is unlikely that one single-institution trial will achieve that objective. We are concerned not only that the Chang publication may influence clinical management in an inappropriate way, but also that it may adversely affect accrual to other clinical trials. One trial that could potentially be affected is an international, randomized, controlled study investigating the role of WBRT following local treatment of brain metastases from melanoma. ${ }^{14}$ This is a collaborative trial being undertaken by the Australia and New Zealand Melanoma Trials Group (ANZMTG) and the Trans-Tasman Radiation Oncology Group (TROG), which commenced patient accrual in December 2008 (ClinicalTrials.gov Identifier: NCT01503827.

Brain metastases from melanoma are a common and serious problem. ${ }^{15-17}$ However, current management practices for melanoma brain metastases are based mainly on studies in other malignancies. ${ }^{13,18,19}$ The role of adjuvant WBRT following surgical resection of cerebral melanoma metastases or their treatment with SRS is controversial. As far as we are aware, the ANZMTG/TROG study is the first randomized, clinical trial in this scenario. Satisfactory patient accrual to this study is critical to its successful completion, and efforts are therefore being made to encourage patient recruitment and enlist more trial sites. The trial is Australian-based, but several European centers (in Norway and the United Kingdom) also are active trial sites. The first trial site in the United States is about to open, although the trial has met significant resistance in that country, where there is a strong preference for SRS alone (even though there are no randomized trial data in melanoma patients). The ethics committee of one of the largest hospitals in Australia, a country that has the highest per capita incidence of melanoma in the world, delayed trial approval citing the Chang publication as evidence that WBRT involves a risk of long-term neurocognitive effects (but eventually gave its approval when the details of the Chang trial, as outlined above and discussed further below, were explained).

Another reason that the report of the Chang trial should not be used to delay the ANZMTG/TROG trial is because the patient populations are quite different. More than half the patients in the Chang trial were lung cancer patients, who can have cognitive impairment not only from their tumor but also from cerebrovascular effects of smoking. Other patients had cerebral metastases from breast cancer, and such patients often have had highly toxic chemotherapy, which can impair neurocognitive function. Most importantly, the endpoints of the two trials differ. The primary endpoint of the ANZMTG/TROG trial of WBRT is distant intracranial disease control, which was actually significantly improved in the Chang trial. Detailed assessment of neurocognitive function in the ANZMTG/TROG study will be performed at the baseline visit, then every 
2 months until distant intracranial failure or death, and is therefore likely to provide a definitive answer to the question of whether long-term neurocognitive function is in fact significantly impaired in melanoma patients treated with WBRT. The need to complete the ANZMTG/TROG trial has been heightened by the recent confirmation, reported at plenary sessions of both ASTRO and ASCO, of the sensitivity of microscopic melanoma metastases to radiotherapy in a randomized trial of adjuvant radiotherapy after lymphadenectomy for macroscopic nodal metastases. ${ }^{20}$

When the results of a trial are not conclusive, it may nevertheless be useful to regard the study as hypothesisgenerating. We suggest that this should be the way in which the Chang trial should be viewed, and the independent Trial Management Committee of the ANZMTG/ TROG study was of the same opinion. In its response to the ethics committees and IRBs of participating centers, the Management Committee emphasized the importance of confirming or refuting the Chang trial results in a multicenter, randomized setting. The ANZMTG/TROG trial should clarify the benefits and risks of adjuvant WBRT for melanoma patients with cerebral metastases.

We believe that major changes to treatment paradigms should not be made on the basis of possibly flawed studies or on the basis of superficial assessment and hasty interpretation of trial results. This is particularly important in diseases where there are few data, such as melanoma. Such restraint also is important when well-designed trials aiming to answer fundamental questions in the relevant area need to be done expeditiously, not delayed. Nor should media publicity about clinical trial outcomes be accepted without critical evaluation of the results that are actually reported.

\section{REFERENCES}

1. Kaplan NM. Recent clinical trials: the good, the bad, and the misleading. Hypertension. 2008;52(4):608-9.

2. Giuliano AE, Hunt KK, Ballman KV, Beitsch PD, Whitworth $\mathrm{PW}$, Blumencranz PW, et al. Axillary dissection vs no axillary dissection in women with invasive breast cancer and sentinel node metastasis: a randomized clinical trial. JAMA. 2011;305 (6):569-75.

3. Grady D. Lymph node study shakes pillar of breast cancer care. New York Times. 2011 February 8, 2011.

4. Chang EL, Wefel JS, Hess KR, Allen PK, Lang FF, Kornguth DG, et al. Neurocognition in patients with brain metastases treated with radiosurgery or radiosurgery plus whole-brain irradiation: a randomised controlled trial. Lancet Oncol. 2009;10 (11): $1037-44$.
5. Sydes MR, Langley RE. Potential pitfalls in the design and reporting of clinical trials. Lancet Oncol. 2010;11(7):694-700.

6. Cranmer LD, Jeter JM, Morgan SS, Hersh EM, Stea B. Wholebrain radiation therapy in melanoma: an open question. Lancet Oncol. 2010;11(1):13-4.

7. Mahmood U, Kwok Y, Regine WF, Patchell RA. Whole-brain irradiation for patients with brain metastases: still the standard of care. Lancet Oncol. 2010;11(3):221-3.

8. Weiss SE, Kelly PJ. Neurocognitive function after WBRT plus SRS or SRS alone. Lancet Oncol. 2010;11(3):220-1.

9. Randomized trial comparing radiosurgery with vs without whole brain radiotherapy [Internet]. October 2011; Available at: http:// clinicaltrials.gov/ct2/show/record/NCT00548756. Accessed 21 Nov 2011.

10. Chan AW, Hrobjartsson A, Haahr MT, Gotzsche PC, Altman DG. Empirical evidence for selective reporting of outcomes in randomized trials: comparison of protocols to published articles. JAMA. 2004;291(20):2457-65.

11. Sanghera P, Gardner SL, Scora D, Davey P. Early expansion of the intracranial CSF volume after palliative whole-brain radiotherapy: results of a longitudinal CT segmentation analysis. Int J Radiat Oncol Biol Phys. 2009;76(4):1171-6.

12. Nelson R. Whole-brain radiation increases risk for cognitive decline in patients with brain metastases. Medscape Medical News: Medscape, LLC; 2009.

13. Aoyama H, Shirato $H$, Tago $M$, Nakagawa $K$, Toyoda $T$, Hatano K, et al. Stereotactic radiosurgery plus whole-brain radiation therapy vs stereotactic radiosurgery alone for treatment of brain metastases: a randomized controlled trial. JAMA. 2006;295(21): 2483-91.

14. Fogarty G, Morton RL, Vardy J, Nowak AK, Mandel C, Forder $\mathrm{PM}$, et al. Whole brain radiotherapy after local treatment of brain metastases in melanoma patients - a randomised phase III trial. BMC Cancer. 2011;11:142.

15. Bafaloukos D, Gogas H. The treatment of brain metastases in melanoma patients. Cancer Treat Rev. 2004;30(6):515-20.

16. Posner JB. Management of brain metastases. Rev Neurol (Paris). 1992;148(6-7):477-87.

17. Sampson JH, Carter JH Jr, Friedman AH, Seigler HF. Demographics, prognosis, and therapy in 702 patients with brain metastases from malignant melanoma. J Neurosurg. 1998;88(1): $11-20$.

18. Fisher B, Seiferheld W, Schultz C, DeAngelis L, Nelson D, Schold SC, et al. Secondary analysis of Radiation Therapy Oncology Group study (RTOG) 9310: an intergroup phase II combined modality treatment of primary central nervous system lymphoma. J Neurooncol. 2005;74(2):201-5.

19. Patchell RA, Tibbs PA, Regine WF, Dempsey RJ, Mohiuddin M, Kryscio RJ, et al. Postoperative radiotherapy in the treatment of single metastases to the brain: a randomized trial. JAMA. 1998;280(17):1485-9.

20. Henderson MA, Burmeister B, Thompson JF, di Iulio J, Fisher R, Hong A, et al. Adjuvant radiotherapy and regional lymph node field control in melanoma patients after lymphadenectomy: results of an intergroup randomized trial (ANZMTG 01.02/ TROG 02.01) [abstract LBA9084]. J Clin Oncol. 2009;27 (Suppl):18s. 